\title{
Periodic Solutions of Second Order Nonlinear Difference Equations with Singular $\phi$-Laplacian Operator
}

\author{
Ruyun Ma and Yanqiong Lu \\ Department of Mathematics, Northwest Normal University, Lanzhou 730070, China \\ Correspondence should be addressed to Ruyun Ma; ruyun_ma@126.com
}

Received 10 April 2014; Accepted 29 June 2014; Published 10 July 2014

Academic Editor: Irena Rachůnková

Copyright (c) 2014 R. Ma and Y. Lu. This is an open access article distributed under the Creative Commons Attribution License, which permits unrestricted use, distribution, and reproduction in any medium, provided the original work is properly cited.

We obtain some existence results of solutions for discrete periodic boundary value problems with singular $\phi$-Laplacian operator $\nabla\left(\Delta u_{k} / \sqrt{1-\kappa\left(\Delta u_{k}\right)^{2}}\right)+r_{k} u_{k}+\left(m_{k} /\left(u_{k}\right)^{\lambda}\right)=e_{k}, 2 \leq k \leq N-1, u_{1}=u_{N}$, and $\Delta u_{1}=\Delta u_{N-1}$ by using the upper and lower solutions method and Brouwer degree theory, where $\kappa>0$ is a constant, $\mathbf{r}=\left(r_{2}, \ldots, r_{N-1}\right), \mathbf{m}=\left(m_{2}, \ldots, m_{N-1}\right), \mathbf{e}=\left(e_{2}, \ldots, e_{N-1}\right) \in \mathbb{R}^{N-2}$, and $\lambda>0$ is a parameter. We also give some examples with singular nonlinearities to illustrate our main results.

\section{Introduction}

In this paper we present some existence results for the discrete periodic boundary value problems with singular $\phi$-Laplacian operator

$$
\begin{gathered}
\nabla\left(\frac{\Delta u_{k}}{\sqrt{1-\kappa\left(\Delta u_{k}\right)^{2}}}\right)+r_{k} u_{k}+\frac{m_{k}}{\left(u_{k}\right)^{\lambda}}=e_{k}, \\
k \in[2, N-1]_{\mathbb{Z}}, \\
u_{1}=u_{N}, \quad \Delta u_{1}=\Delta u_{N-1},
\end{gathered}
$$

where $\kappa>0$ is a constant, $\mathbf{r}=\left(r_{2}, \ldots, r_{N-1}\right), \mathbf{m}=\left(m_{2}, \ldots\right.$, $\left.m_{N-1}\right), \mathbf{e}=\left(e_{2}, \ldots, e_{N-1}\right) \in \mathbb{R}^{N-2}, \lambda>0$ is a parameter, and $[2, N-1]_{\mathbb{Z}}:=\{2,3, \ldots, N-1\}$ with $N>4$ is an integer.

These problems are originated in the study that a particle moves on a straight line, subject to a restoring force $F$ with relativistic effects, which can be described by the differential equation of motion:

$$
\left(\frac{m_{0} u^{\prime}}{\sqrt{1-u^{\prime 2} / c^{2}}}\right)^{\prime}=F,
$$

where $m_{0}$ is the rest mass of the particle and $c$ is the speed of light in the vacuum (see [1-3]). Assume that $m_{0}=c=1$.
The existence and multiplicity of solutions for (2) subjected to Dirichlet, Robin, periodic, or Neumann boundary conditions have been studied by various methods, such as the method of lower and upper solutions, topological degree theory, and critical point theory; see [4-8] and the references therein.

An interesting question is which techniques and theorems regarding the continuous differential equations can be adapted for difference equations (see Kelly and Peterson [9], Agarwal [10], and Bereanu and Mawhin [11, 12]). The purpose of this paper is to show that some known existence and multiplicity results of periodic solution for singular perturbations of the singular $\phi$-Laplacian operator also hold for the corresponding difference equation and develop some results for the singular difference equation boundary value problems; see [12-17].

In the case $\kappa=1$ and $\mathbf{r}=0$, Bereanu and Mawhin [12] proved that the discrete periodic problem with an attractive nonlinearity

$$
\begin{gathered}
\nabla\left(\frac{\Delta u_{k}}{\sqrt{1-\left(\Delta u_{k}\right)^{2}}}\right)+\frac{1}{\left(u_{k}\right)^{\lambda}}=e_{k}, \quad k \in[2, N-1]_{\mathbb{Z}} \\
u_{1}=u_{N}, \quad \Delta u_{1}=\Delta u_{N-1}
\end{gathered}
$$


has at least one positive solution if and only if $\overline{\mathbf{e}}=1 /(\mathrm{N}-$ 2) $\sum_{s=2}^{N-1} e_{s}>0$. When $\lambda \geq 1$, they also showed that the repulsive singular periodic problem

$$
\begin{gathered}
\nabla\left(\frac{\Delta u_{k}}{\sqrt{1-\left(\Delta u_{k}\right)^{2}}}\right)-\frac{1}{\left(u_{k}\right)^{\lambda}}=e_{k}, \quad k \in[2, N-1]_{\mathbb{Z}} \\
u_{1}=u_{N}, \quad \Delta u_{1}=\Delta u_{N-1}
\end{gathered}
$$

has at least one positive solution if and only if $\overline{\mathbf{e}}<0$.

In the case $\kappa=0$, the problem (1) is the classical discrete periodic problem

$$
\begin{gathered}
\nabla\left(\Delta u_{k}\right)+r_{k} u_{k}+\frac{m_{k}}{\left(u_{k}\right)^{\lambda}}=e_{k}, \quad k \in[2, N-1]_{\mathbb{Z}}, \\
u_{1}=u_{N}, \quad \Delta u_{1}=\Delta u_{N-1} .
\end{gathered}
$$

This problem has been studied by Ma and Lu [15-17] where the existence of positive solution needs a necessary condition $r_{k}<0$ or $0<r_{k}<4 \sin ^{2}(\pi / 2 N)$ (see [14]). For other results concerning the existence of solutions for singular nonlinear difference equation boundary value problems, see, for example, $[9,10,18]$.

It is interesting to remark that, in contrast to the classical case, the periodic problem with discrete relativistic acceleration

$$
\begin{gathered}
\nabla\left(\frac{\Delta u_{k}}{\sqrt{1-\left(\Delta u_{k}\right)^{2}}}\right)+r u_{k}=e_{k}, \quad k \in[2, N-1]_{\mathbb{Z}} \\
u_{1}=u_{N}, \quad \Delta u_{1}=\Delta u_{N-1}
\end{gathered}
$$

has at least one solution for any $r \neq 0$ and any forcing term $\mathbf{e}$ (see [12, Corollary 2]). Note that, for this type of problems, in some sense, the same situation occurs also if we add a singular nonlinearity.

In order to explain the main result, let us introduce some notation. For any $x \in \mathbb{R}$, we write $x^{+}=\max \{x, 0\}$ and $x^{-}=$ $\max \{-x, 0\}$. For $\mathbf{e}=\left(e_{2}, \ldots, e_{N-1}\right) \in \mathbb{R}^{N-2}$, we put

$$
E=\sum_{k=2}^{N-1} e_{k}, \quad E_{ \pm}=\sum_{k=2}^{N-1} e_{k}^{ \pm}
$$

and note that $E=E_{+}-E_{-}$.

Motivated by the above results from [12, 14-17], we consider the discrete periodic problem

$$
\begin{gathered}
\nabla\left(\frac{\Delta u_{k}}{\sqrt{1-\kappa\left(\Delta u_{k}\right)^{2}}}\right)+r_{k} u_{k}-\frac{1}{\left(u_{k}\right)^{\lambda}}=e_{k}, \\
k \in[2, N-1]_{\mathbb{Z}}, \\
u_{1}=u_{N}, \quad \Delta u_{1}=\Delta u_{N-1},
\end{gathered}
$$

where $\mathbf{r}=\left(r_{2}, \ldots, r_{N-1}\right), \mathbf{e}=\left(e_{2}, \ldots, e_{N-1}\right) \in \mathbb{R}^{N-2}$, and $\lambda \geq$ 1. If either $\overline{\mathbf{r}}>0$ or $\overline{\mathbf{r}}=0$ and $\overline{\mathbf{e}}<-N R_{-} / 2 \sqrt{\kappa}(N-2)$, then we prove that the above problem has at least one solution (see Example 14). In the case $\overline{\mathbf{r}}<0$, we show that the periodic problem with repulsive singular term

$$
\begin{gathered}
\nabla\left(\frac{\Delta u_{k}}{\sqrt{1-\kappa\left(\Delta u_{k}\right)^{2}}}\right)+r_{k} u_{k}-\frac{m_{k}}{\left(u_{k}\right)^{\lambda}}=e_{k}, \\
k \in[2, N-1]_{\mathbb{Z}}, \\
u_{1}=u_{N}, \quad \Delta u_{1}=\Delta u_{N-1},
\end{gathered}
$$

with $\lambda>0$ and $\mathbf{m}=\left(m_{2}, \ldots, m_{N-1}\right) \geq 0$, is solvable (see Example 18) provided that

$$
-E>(1+\lambda)\left[\frac{|R|^{\lambda} M}{\lambda^{\lambda}}\right]^{1 /(1+\lambda)}+\frac{N R_{-}}{2 \sqrt{\kappa}} .
$$

On the other hand, we also consider the periodic problem with attractive singularity

$$
\begin{gathered}
\nabla\left(\frac{\Delta u_{k}}{\sqrt{1-\kappa\left(\Delta u_{k}\right)^{2}}}\right)+r_{k} u_{k}+\frac{m_{k}}{\left(u_{k}\right)^{\lambda}}=e_{k}, \\
k \in[2, N-1]_{\mathbb{Z}}, \\
u_{1}=u_{N}, \quad \Delta u_{1}=\Delta u_{N-1},
\end{gathered}
$$

where $\mathbf{r}=\left(r_{2}, \ldots, r_{N-1}\right), \mathbf{m}=\left(m_{2}, \ldots, m_{N-1}\right)$, and $\mathbf{e}=\left(e_{2}\right.$, $\left.\ldots, e_{N-1}\right) \in \mathbb{R}^{N-2}$ with $\mathbf{m} \geq 0$ and $\lambda>0$. If either $\overline{\mathbf{r}}<0$ or $\mathbf{r}=0$ and in both cases $E<M(2 \sqrt{\kappa} / N)^{\lambda}-\left(N R_{-} / 2 \sqrt{\kappa}\right)$, then the problem (12) has at least one solution (see Example 19). Moreover, in the pure attractive case, that is, $\mathbf{m}>0$, it follows that (12) is solvable if either $\overline{\mathbf{r}}<0$ or $\overline{\mathbf{r}}=0$ and $E>N R_{+} / 2 \sqrt{\kappa}$ (Theorem 21).

The rest of the paper is organized as follows. In Section 2, we introduce some notations and auxiliary results. In Section 3 we establish the method of non-well-ordered lower and upper solutions and give an application on the discrete periodic problem with the strong repulsive nonlinearities. In addition, we introduce some methods to construct lower and upper solutions. Finally, in Section 4 we give some applications to deal with the singular perturbations periodic problems.

\section{Some Notations and Auxiliary Results}

In this section, we first introduce some notations. Let $a, b \in \mathbb{N}$ with $a<b$; we denote $[a, b]_{\mathbb{Z}}:=\{a, a+1, \ldots, b\}$.

For $\mathbf{u}=\left(u_{1}, \ldots, u_{p}\right) \in \mathbb{R}^{p}$, set $\|\mathbf{u}\|_{\infty}=\max _{1 \leq k \leq p}\left|u_{k}\right|$, $\|\mathbf{u}\|_{1}=\sum_{k=1}^{p}\left|u_{k}\right|$. If $\alpha, \beta \in \mathbb{R}^{p}$, we write $\alpha \leq \beta$ (resp., $\alpha<\beta$ ) if $\alpha_{k} \leq \beta_{k}$ (resp., $\alpha_{k}<\beta_{k}$ ) for all $1 \leq k \leq p$. The following assumption upon $\phi$ (called singular) is made throughout the paper:

$\left(H_{\phi}\right) \phi:(-a, a) \rightarrow \mathbb{R}(0<a<\infty)$ is an increasing homeomorphism with $\phi(0)=0$.

The model example is

$$
\phi(s)=\frac{s}{\sqrt{1-\kappa s^{2}}}, \quad s \in\left(-\frac{1}{\sqrt{\kappa}}, \frac{1}{\sqrt{\kappa}}\right) .
$$


Let $N \in \mathbb{N}$ with $N \geq 4$ be fixed and $\mathbf{u}=\left(u_{1}, u_{2}, \ldots, u_{N}\right) \in$ $\mathbb{R}^{N}$. Then we denote

$$
\Delta \mathbf{u}=\left(\Delta u_{1}, \ldots, \Delta u_{N-1}\right) \in \mathbb{R}^{N-1},
$$

where $\Delta u_{k}=u_{k+1}-u_{k}$ for $k \in[1, N-1]_{\mathbb{Z}}$ and if $\|\Delta \mathbf{u}\|_{\infty}:=$ $\max _{k \in[1, N-1]_{Z}}\left|\Delta u_{k}\right|<a$, define

$$
\nabla[\phi(\Delta \mathbf{u})]=\left(\nabla\left[\phi\left(\Delta u_{2}\right)\right], \ldots, \nabla\left[\phi\left(\Delta u_{N-1}\right)\right]\right) \in \mathbb{R}^{N-2},
$$

where $\nabla\left[\phi\left(\Delta u_{k}\right)\right]=\phi\left(\Delta u_{k}\right)-\phi\left(\Delta u_{k-1}\right)$ for $k \in[2, N-1]_{\mathbb{Z}}$.

Let $f:[2, N-1]_{\mathbb{Z}} \times \mathbb{R}^{2} \rightarrow \mathbb{R}$ be a continuous function. Then its Nemytskii operator $N_{f}(\mathbf{u}): \mathbb{R}^{N} \rightarrow \mathbb{R}^{N-2}$ is given by

$$
N_{f}(\mathbf{u})=\left(f\left(2, u_{2}, \Delta u_{2}\right), \ldots, f\left(N-1, u_{N-1}, \Delta u_{N-1}\right)\right) .
$$

It follows that $N_{f}$ is continuous and takes bounded sets into bounded sets.

Let $Q$ be the projectors defined by

$$
\overline{\mathbf{u}}=Q \mathbf{u}=\frac{1}{N-2} \sum_{k=2}^{N-1} u_{k} \quad \forall \mathbf{u} \in \mathbb{R}^{N} .
$$

If $\mathbf{u} \in \mathbb{R}^{N}$, we write $\widetilde{\mathbf{u}}=\mathbf{u}-\overline{\mathbf{u}}$ and we will consider the following closed subspaces of $\mathbb{R}^{N}$ :

$$
V^{N-2}=\left\{\mathbf{u} \in \mathbb{R}^{N} \mid u_{1}=u_{N}, \Delta u_{1}=\Delta u_{N-1}\right\} .
$$

Let the vector space $V^{N-2}$ be endowed with the orientation of $\mathbb{R}^{N}$ and the norm $\|\mathbf{u}\|_{\infty}=\max _{1 \leq k \leq N}\left|u_{k}\right|$. Its elements can be associated with the coordinates $\left(u_{2}, \ldots, u_{N-1}\right)$ and correspond to the elements of $\mathbb{R}^{N}$ of the form

$$
\left(\frac{u_{2}+u_{N-1}}{2}, u_{2}, u_{3}, \ldots, u_{N-1}, \frac{u_{2}+u_{N-1}}{2}\right) .
$$

Now, we recall the following technical result given as Lemma 1 from [12].

Lemma 1. For each $\mathbf{h}=\left(h_{2}, \ldots, h_{N-1}\right)$, there exists a unique $\gamma:=Q_{\phi}(\mathbf{h})$ such that

$$
2 \phi^{-1}(\gamma)+\sum_{k=3}^{N-1} \phi^{-1}\left(\sum_{j=2}^{k-1} h_{j}+\gamma\right)=0 .
$$

Moreover, the function $Q_{\phi}$ is continuous.

Lemma 2. Let $F: \mathbb{R}^{N} \rightarrow \mathbb{R}^{N-2}$ be a continuous operator which takes bounded sets into bounded sets and consider the abstract discrete periodic problem:

$$
\nabla[\phi(\Delta \mathbf{u})]=F(\mathbf{u}), \quad u_{1}=u_{N}, \quad \Delta u_{1}=\Delta u_{N-1} .
$$

A function $\mathbf{u}$ is a solution of (21) if and only if $\mathbf{u} \in V^{N-2}$ is a fixed point of the continuous operator $\mathscr{A}_{F}: V^{N-2} \rightarrow V^{N-2}$ defined by $\mathscr{A}_{F}(\mathbf{u})=\mathbf{v}$, where $\mathbf{v}=\left(v_{1}, v_{2}, \ldots, v_{N}\right) \in V^{N-2}$ satisfying

$$
\begin{aligned}
v_{2}= & u_{2}+Q N_{F}(\mathbf{u}), \\
v_{k}= & u_{2}+Q N_{F}(\mathbf{u}) \\
& +\sum_{j=3}^{k} \phi^{-1}\left(\sum_{l=2}^{j-1} F\left(u_{k}\right)+Q_{\phi}\left(N_{F}(\mathbf{u})\right)\right), \\
& \quad k \in[3, N-1]_{\mathbb{Z}} .
\end{aligned}
$$

Furthermore, $\|\Delta(\mathscr{A}(\mathbf{u}))\|_{\infty}<$ a for all $\mathbf{u} \in V^{N-2}$ and

$$
\|\tilde{\mathbf{u}}\|_{\infty}<a(N-2)
$$

for any solution $\mathbf{u}$ of (21).

Let us consider the discrete periodic problem

$$
\begin{aligned}
& \nabla\left[\phi\left(\Delta u_{k}\right)\right]=f\left(k, u_{k}, \Delta u_{k}\right), \\
& u_{1}=u_{N}, \quad \Delta u_{1}=\Delta u_{N-1} .
\end{aligned}
$$

Obviously, from Lemma 2, the fixed point operator associated with (24) is

$$
\mathscr{A}_{f}(\mathbf{u})=\mathbf{u}
$$

Now, we state the method of upper and lower solutions for discrete periodic problem (24) according to Bereanu and Mawhin [12].

Definition 3. A function $\alpha=\left(\alpha_{1}, \ldots, \alpha_{N}\right)$ (resp., $\beta=$ $\left.\left(\beta_{1}, \ldots, \beta_{N}\right)\right)$ is called a lower solution (resp., an upper solution) for (24) if $\|\Delta \alpha\|_{\infty}<a$ (resp., $\|\Delta \beta\|_{\infty}<a$ ) and

$$
\begin{aligned}
& \nabla\left[\phi\left(\Delta \alpha_{k}\right)\right] \geq f\left(k, \alpha_{k}, \Delta \alpha_{k}\right), \\
& \left(\text { resp. } \nabla\left[\phi\left(\Delta \beta_{k}\right)\right] \leq f\left(k, \beta_{k}, \Delta \beta_{k}\right)\right), \\
& k \in[2, N-1]_{\mathbb{Z}}, \\
& \alpha_{1}=\alpha_{N}, \quad \Delta \alpha_{1} \geq \Delta \alpha_{N-1}, \\
& \text { (resp. } \left.\beta_{1}=\beta_{N}, \Delta \beta_{1} \leq \Delta \beta_{N-1}\right) .
\end{aligned}
$$

Such a lower or an upper solution is called strict if the inequality (26) is strict.

Lemma 4 (see [12, Theorem 3]). If (24) has a lower solution $\alpha$ and an upper solution $\beta$ such that $\alpha \leq \beta$, then (24) has a solution $\mathbf{u}$ such that $\alpha \leq \mathbf{u} \leq \beta$. Moreover, if $\alpha$ and $\beta$ are strict, then $\alpha<\mathbf{u}<\beta$, and

$$
\operatorname{deg}\left[I-\mathscr{A}_{f}, \Omega_{\alpha, \beta}, \mathbf{0}\right]=-1,
$$

where $\Omega_{\alpha, \beta}=\left\{\mathbf{u} \in V^{N-2} \mid \alpha<\mathbf{u}<\beta,\|\Delta \mathbf{u}\|_{\infty}<a\right\}$.

An easy adaption of the proof of [12, Theorem 3] provides the following useful result. 
Lemma 5. Assume that (24) has a lower solution $\alpha$ and an upper solution $\beta$ such that $\alpha<\beta$, and

$$
\mathbf{u} \neq \mathscr{A}_{f}(\mathbf{u}) \quad \forall u \in \partial \Omega_{\alpha, \beta} .
$$

Then

$$
\operatorname{deg}\left[I-\mathscr{A}_{f}, \Omega_{\alpha, \beta}, \mathbf{0}\right]=-1 .
$$

The next result is an elementary estimation of the oscillation of a periodic function.

Lemma 6. If $u: \mathbb{Z} \rightarrow \mathbb{R}$ is a $N$-periodic function, then

$$
\max _{k \in[1, N]_{\mathbb{Z}}} u_{k}-\min _{k \in[1, N]_{\mathbb{Z}}} u_{k} \leq \frac{N}{2}\|\Delta \mathbf{u}\|_{\infty} .
$$

Proof. Let $k_{*} \in[1, N-1]_{\mathbb{Z}}$ be such that $u_{k_{*}}=\min _{k \in[1, N]_{\mathbb{Z}}} u_{k}$, and let $k^{*} \in\left[k_{*}, k_{*}+N\right]_{\mathbb{Z}}$ be such that $u_{k^{*}}=\max _{k \in[1, N]_{\mathbb{Z}}} u_{k}$. We have that

$$
\begin{gathered}
u_{k^{*}}-u_{k_{*}}=\sum_{s=k_{*}}^{k^{*}-1} \Delta u_{s} \leq\left(k^{*}-k_{*}\right)\|\Delta \mathbf{u}\|_{\infty}, \\
u_{k^{*}}-u_{k_{*}}=\sum_{s=k^{*}}^{k_{*}+N-1}\left(-\Delta u_{s}\right) \leq\left(k_{*}+N-k^{*}\right)\|\Delta \mathbf{u}\|_{\infty} .
\end{gathered}
$$

Then, multiplying both inequalities and using that $x y \leq(x+$ $y)^{2} / 2$, for all $x, y \in \mathbb{R}$, it follows that

$$
\left(u_{k^{*}}-u_{k_{*}}\right)^{2} \leq \frac{\left[N\|\Delta \mathbf{u}\|_{\infty}\right]^{2}}{4},
$$

and the proof is completed.

\section{The Method of Lower and Upper Solutions and Application}

In 2008, Bereanu and Mawhin [12] proved that problem (24) has at least one solution if it has a lower solution $\alpha$ and an upper solution $\beta$ with $\alpha \leq \beta$. In the following result we prove some additional concerning the location of the solution. In particular, we have a posteriori estimations which will be very useful in the sequel (Remark 8).

Theorem 7. Assume that (24) has a lower solution $\alpha$ and an upper solution $\beta$ such that

$$
\exists k_{\star} \in[1, N]_{\mathbb{Z}}: \alpha_{k_{\star}}>\beta_{k_{\star}} .
$$

Then (24) has at least one solution $\mathbf{u}$ such that

$$
\begin{array}{r}
\min \left\{\alpha_{k_{u}}, \beta_{k_{u}}\right\} \leq u_{k_{u}} \leq \max \left\{\alpha_{k_{u}}, \beta_{k_{u}}\right\} \\
\text { for some } k_{u} \in[1, N]_{\mathbb{Z}} .
\end{array}
$$

Proof. Let

$$
\begin{aligned}
u^{*}= & \|\alpha\|_{\infty}+\|\beta\|_{\infty}+a(N-2), \\
m=\max & \{|f(k, u, v)|+1 \mid \\
& (k, u, v) \in[2, N-1]_{\mathbb{Z}} \\
& \left.\times\left[-u^{*}-2, u^{*}+2\right] \times[-a, a]\right\},
\end{aligned}
$$

and define the continuous function $g:[2, N-1]_{\mathbb{Z}} \times \mathbb{R}^{2} \rightarrow \mathbb{R}$ by

$$
\begin{aligned}
& g(k, u, v) \\
& = \begin{cases}-m-1, & u \leq-u^{*}-1, \\
f(k, u, v)+\left(u+u^{*}\right) & \\
\quad \times(m+1+f(k, u, v)), & -u^{*}-1<u<-u^{*}, \\
f(k, u, v), & -u^{*} \leq u \leq u^{*}, \\
f(k, u, v)+\left(u-u^{*}\right) m, & u^{*}<u<u^{*}+1, \\
f(k, u, v)+m, & u \geq u^{*}+1 .\end{cases}
\end{aligned}
$$

Let us consider the modified periodic problem

$$
\begin{gathered}
\nabla\left[\phi\left(\Delta u_{k}\right)\right]=g\left(k, u_{k}, \Delta u_{k}\right), \\
u_{1}=u_{N}, \quad \Delta u_{1}=\Delta u_{N-1},
\end{gathered}
$$

and let $\mathscr{A}_{g}$ be the fixed point operator associated with (37).

It is not difficult to verify that $\alpha$ is a lower solution and $\beta$ is an upper solution of the problem (37). Moreover, by computation, $\alpha_{1}=-u^{*}-2$ is a lower solution of (37) and $\beta_{1}=u^{*}+2$ is an upper solution of (37). Notice that

$$
\alpha_{1}<\min \{\alpha, \beta\} \leq \max \{\alpha, \beta\}<\beta_{1},
$$

which together with (33) imply that

$$
\Omega_{\alpha_{1}, \beta} \cup \Omega_{\alpha, \beta_{1}} \subset \Omega_{\alpha_{1}, \beta_{1}}, \quad \Omega_{\alpha_{1}, \beta} \cap \Omega_{\alpha, \beta_{1}}=\emptyset .
$$

So, we can consider the open bounded set

$$
\Omega=\Omega_{\alpha_{1}, \beta_{1}} \backslash\left[\overline{\Omega_{\alpha_{1}, \beta}} \cup \overline{\Omega_{\alpha, \beta_{1}}}\right] .
$$

It follows that

$$
\begin{aligned}
\Omega= & \left\{u \in \Omega_{\alpha_{1}, \beta_{1}} \mid\right. \\
& u_{k_{u}}>\beta_{k_{u}}, \\
& \left.u_{s_{u}}<\alpha_{s_{u}} \text { for some } k_{u}, s_{u} \in[1, N]_{\mathbb{Z}}\right\}, \\
& \partial \Omega=\partial \Omega_{\alpha_{1}, \beta_{1}} \cup \partial \Omega_{\alpha_{1}, \beta} \cup \partial \Omega_{\alpha, \beta_{1}} .
\end{aligned}
$$

Clearly, any constant function between $\beta_{k_{\star}}$ and $\alpha_{k_{\star}}$ is contained in $\Omega$, so $\Omega \neq \emptyset$.

Next, let us consider $\mathbf{u} \in \partial \Omega$ such that $\mathscr{A}_{g}(\mathbf{u})=\mathbf{u}$ and $\|\mathbf{u}\|_{\infty}=u^{*}+2$. Notice that one has $\|\Delta \mathbf{u}\|_{\infty}<a$. This implies that there exists $k_{0} \in[1, N]_{\mathbb{Z}}$ such that $u_{k_{0}}=\max _{k \in[1, N]_{\mathbb{Z}}} u_{k}=$ $u^{*}+2$ or $u_{k_{0}}=\min _{k \in[1, N]_{Z}} u_{k}=-u^{*}-2$. In the first case we can assume that $k_{0} \in[1, N-1]_{\mathbb{Z}}$. If $k_{0} \in[2, N-1]_{\mathbb{Z}}$, then $\Delta u_{k_{0}} \leq 0, \Delta u_{k_{0}-1} \geq 0$. This together with $\phi$ is an increasing homeomorphism implying $\nabla\left[\phi\left(\Delta u_{k_{0}}\right)\right] \leq 0$. On the other hand, we have that

$$
\nabla\left[\phi\left(\Delta u_{k_{0}}\right)\right]=f\left(k_{0}, u_{k_{0}}, \Delta u_{k_{0}}\right)+m>0,
$$

which is a contradiction. If $k_{0}=1$, then from boundary condition $u_{1}=u_{N}, \Delta u_{1}=\Delta u_{N-1}$, we can get that $\Delta u_{1} \leq 0$ 
and $\Delta u_{N-1} \geq 0$, which implies that $\Delta u_{1}=\Delta u_{N-1}=0$. This together with $\nabla\left[\phi\left(\Delta u_{N-1}\right)\right]=\phi\left(\Delta u_{N-1}\right)-\phi\left(\Delta u_{N-2}\right)=f(N-$ $\left.1, u_{N-1}, 0\right)+m>0$ implies that $u_{N}=u_{N-1}<u_{N-2}$; this is a contradiction. Analogously, one can obtain a contradiction in the second case. Consequently,

$$
\left[\mathbf{u} \in \partial \Omega, \mathscr{A}_{g}(\mathbf{u})=\mathbf{u}\right] \Longrightarrow\|\mathbf{u}\|_{\infty}<u^{*}+2
$$

Now, let $\mathbf{u} \in \partial \Omega$ be such that $\mathscr{A}_{g}(\mathbf{u})=\mathbf{u}$. It follows from (43) that $\|\mathbf{u}\|_{\infty}<u^{*}+2,\|\Delta \mathbf{u}\|_{\infty}<a$ and $\mathbf{u} \in \partial \Omega_{\alpha_{1}, \beta} \cup \partial \Omega_{\alpha, \beta_{1}}$. We infer that there exists $k_{0} \in[1, N]_{\mathbb{Z}}$ such that $u_{k_{0}}=\alpha_{k_{0}}$ or $u_{k_{0}}=\beta_{k_{0}}$, implying that $\left|u_{k_{0}}\right| \leq\|\alpha\|_{\infty}+\|\beta\|_{\infty}$. Then,

$$
\left|u_{k}\right| \leq\left|u_{k_{0}}\right|+\sum_{s=2}^{N-1}\left|\Delta u_{s}\right|<u^{*} \quad \forall k \in[1, N]_{\mathbb{Z}},
$$

and, consequently,

$$
\left[\mathbf{u} \in \partial \Omega, \mathscr{A}_{g}(\mathbf{u})=\mathbf{u}\right] \Longrightarrow\|\mathbf{u}\|_{\infty}<u^{*} .
$$

We have divided two cases to discuss.

Case 1. Assume that there exists $\mathbf{u} \in \partial \Omega$ such that $\mathscr{A}_{g}(\mathbf{u})=\mathbf{u}$. Using (45), we deduce that $\|\mathbf{u}\|_{\infty}<u^{*}$, implying that $\mathbf{u}$ is a solution of (24) and (34) holds. Actually, in this case, there exists $k_{u} \in[1, N]_{\mathbb{Z}}$ such that $u_{k_{u}}=\alpha_{k_{u}}$ or $u_{k_{u}}=\beta_{k_{u}}$.

Case 2. Assume that $\mathscr{A}_{g}(\mathbf{u}) \neq \mathbf{u}$ for all $\mathbf{u} \in \partial \Omega$. Then, from Lemma 5 applied to $g$, it follows that

$$
\begin{aligned}
\operatorname{deg}\left[I-\mathscr{A}_{g}, \Omega_{\alpha_{1}, \beta_{1}}, \mathbf{0}\right] & =\operatorname{deg}\left[I-\mathscr{A}_{g}, \Omega_{\alpha_{1}, \beta}, \mathbf{0}\right] \\
& =\operatorname{deg}\left[I-\mathscr{A}_{g}, \Omega_{\alpha, \beta_{1}}, \mathbf{0}\right]=-1 .
\end{aligned}
$$

This together with the additivity property of the Brouwer degree implies that

$$
\operatorname{deg}\left[I-\mathscr{A}_{g}, \Omega, \mathbf{0}\right]=1,
$$

which together with the existence property of the Brouwer degree imply that there exists $\mathbf{u} \in \Omega$ such that $\mathscr{A}_{g}(\mathbf{u})=\mathbf{u}$. It follows that there exists $k_{1}, k_{2} \in[1, N]_{\mathbb{Z}}$ such that $u_{k_{1}}<\alpha_{k_{1}}$ and $u_{k_{2}}>\beta_{k_{2}}$. Then, using once again the fact that $\|\Delta \mathbf{u}\|_{\infty}<$ $a$, it follows that $\|\mathbf{u}\|_{\infty}<u^{*}$ and $\mathbf{u}$ is a solution of (24). Moreover, from $\mathbf{u} \in \Omega$, it follows that (34) is true.

Remark 8. Assume that (24) has a lower solution $\alpha$ and an upper solution $\beta$. From Lemma 4 and Theorem 7 , we deduce that (24) has at least one solution u satisfying (34). In particular,

$$
\|\mathbf{u}\|_{\infty}<\|\alpha\|_{\infty}+\|\beta\|_{\infty}+a(N-2) .
$$

As an application of Theorem 7, we deal with singular strong nonlinearities. Consider the following discrete periodic problem:

$$
\begin{gathered}
\nabla\left[\phi\left(\Delta u_{k}\right)\right]+h\left(u_{k}\right) \Delta u_{k}=g\left(u_{k}\right)+f\left(k, u_{k}, \Delta u_{k}\right), \\
u_{1}=u_{N}, \quad \Delta u_{1}=\Delta u_{N-1},
\end{gathered}
$$

where $f:[2, N-1]_{\mathbb{Z}} \times \mathbb{R}^{2} \rightarrow \mathbb{R}$ and $h, g:(0, \infty) \rightarrow \mathbb{R}$ are continuous functions such that

$$
\lim _{u \rightarrow 0_{+}} g(u)=+\infty
$$

and $h \geq 0$. Under those assumptions we have the following theorem.

Theorem 9. Assume that (49) has a lower solution $\alpha>0$ and an upper solution $\beta>0$. Then (49) has at least one solution $\mathbf{u}$ which satisfies (34).

Proof. First, we define some notations as follows:

$$
\begin{gathered}
\delta=\min _{[1, N]_{\mathbb{Z}}} \min \{\alpha, \beta\}, \quad B=\|\alpha\|_{\infty}+\|\beta\|_{\infty}+a(N-2), \\
m=\min _{[1, N]_{\mathbb{Z}} \times[-B, B] \times[-a, a]} f .
\end{gathered}
$$

From (50), there exists $\varepsilon \in(0, \delta)$ such that

$$
\begin{aligned}
g\left(u_{k}\right)> & h(\varepsilon) \sum_{s \in I_{1}} \Delta u_{s}+\sum_{s \in I_{2}} h\left(u_{s}\right) \Delta u_{s}-(N-2) m \\
& -\sum_{s \in I_{2}} g\left(u_{s}\right), u_{k} \in(0, \varepsilon]
\end{aligned}
$$

where $I_{1}=\left\{k \mid 0<u_{k} \leq \varepsilon\right\}, I_{2}=\left\{k \mid u_{k}>\varepsilon\right\}$.

Let $\widehat{g}, \widehat{h}: \mathbb{R} \rightarrow \mathbb{R}$ be the continuous functions given by

$$
\begin{aligned}
& \widehat{g}(u)= \begin{cases}g(u), & u \geq \varepsilon, \\
g(\varepsilon), & u \leq \varepsilon,\end{cases} \\
& \widehat{h}(u)= \begin{cases}h(u), & u \geq \varepsilon, \\
h(\varepsilon), & u \leq \varepsilon,\end{cases}
\end{aligned}
$$

and consider the auxiliary periodic problem

$$
\begin{gathered}
\nabla\left[\phi\left(\Delta u_{k}\right)\right]+\widehat{h}\left(u_{k}\right) \Delta u_{k}=\widehat{g}\left(u_{k}\right)+f\left(k, u_{k}, \Delta u_{k}\right), \\
u_{1}=u_{N}, \quad \Delta u_{1}=\Delta u_{N-1} .
\end{gathered}
$$

From $\varepsilon<\delta$, it follows that $\alpha$ and $\beta$ are lower and upper solutions of (54), respectively.

If $\alpha \leq \beta$, then (54) has a solution $\mathbf{u}$ satisfying $\alpha \leq \mathbf{u} \leq \beta$ from Lemma 4 and [12, Remark 3] (without any additional assumption). If condition (33) holds, then (54) has a solution u satisfying (34). Obviously, the solution u satisfies

$$
-B \leq \mathbf{u} \leq B, \quad\|\Delta \mathbf{u}\|_{\infty}<a .
$$

Next, we will prove that $\mathbf{u}>\varepsilon$. From (55), there exists $k_{0} \in[1, N]_{\mathbb{Z}}$ such that $u_{k_{0}}=\min _{k \in[1, N]_{\mathbb{Z}}} u_{k}$. Suppose on the contrary that $u_{k_{0}} \leq \varepsilon$, summing from $s=2$ to $s=N-1$ for (54); then we have

$$
\sum_{s=2}^{N-1} \widehat{h}\left(u_{s}\right) \Delta u_{s}=\sum_{s=2}^{N-1} \widehat{g}\left(u_{s}\right)+\sum_{s=2}^{N-1} f\left(s, u_{s}, \Delta u_{s}\right) .
$$


This together with (52) implies that

$$
\begin{aligned}
0= & \sum_{s=2}^{N-1} \widehat{g}\left(u_{s}\right)-\sum_{s=2}^{N-1} \widehat{h}\left(u_{s}\right) \Delta u_{s}+\sum_{s=2}^{N-1} f\left(s, u_{s}, \Delta u_{s}\right) \\
= & \sum_{s \in I_{1}} \widehat{g}\left(u_{s}\right)+\sum_{s \in I_{2}} \widehat{g}\left(u_{s}\right)-\sum_{s=2}^{N-1} \widehat{h}\left(u_{s}\right) \Delta u_{s} \\
& +\sum_{s=2}^{N-1} f\left(s, u_{s}, \Delta u_{s}\right) \\
= & g(\varepsilon) \sum_{s \in I_{1}} 1+\sum_{s \in I_{2}} g\left(u_{s}\right)-h(\varepsilon) \sum_{s \in I_{1}} \Delta u_{s}-\sum_{s \in I_{2}} h\left(u_{s}\right) \Delta u_{s} \\
& +\sum_{s=2}^{N-1} f\left(s, u_{s}, \Delta u_{s}\right) \\
\geq & g(\varepsilon)+\sum_{s \in I_{2}} g\left(u_{s}\right)-h(\varepsilon) \sum_{s \in I_{1}} \Delta u_{s}-\sum_{s \in I_{2}} h\left(u_{s}\right) \Delta u_{s} \\
& +\sum_{s=2}^{N-1} f\left(s, u_{s}, \Delta u_{s}\right)>0,
\end{aligned}
$$

which is a contradiction. Hence, $\mathbf{u}>\varepsilon$, implying that $\mathbf{u}$ is also a solution of (49).

Now, we give a method to construct the lower solution and the upper solution of the following discrete periodic problem:

$$
\begin{aligned}
\nabla\left[\phi\left(\Delta u_{k}\right)\right] & =g_{0}\left(k, u_{k}\right)+e_{k}, \quad k \in[2, N-1]_{\mathbb{Z}} \\
u_{1} & =u_{N}, \quad \Delta u_{1}=\Delta u_{N-1},
\end{aligned}
$$

where $g_{0}:[2, N-1]_{\mathbb{Z}} \times(0, \infty) \rightarrow \mathbb{R}$ is a continuous singular nonlinearity and $\mathbf{e}=\left(e_{2}, \ldots, e_{N-1}\right) \in \mathbb{R}^{N-2}$.

The following result gives a method to construct a lower solution to (58), getting also control on its localization.

Theorem 10. Suppose that there exist $u^{1}>0$ and $\mathbf{c}=$ $\left(c_{2}, \ldots, c_{N-1}\right) \in \mathbb{R}^{N-2}$ such that

$$
g_{0}(k, u) \leq c_{k}, \quad \forall(k, u) \in[2, N-1]_{\mathbb{Z}} \times\left[u^{1}, u^{1}+\frac{a N}{2}\right] .
$$

If

$$
\overline{\mathbf{c}}+\overline{\mathbf{e}} \leq 0
$$

then (58) has a lower solution $\alpha$ such that

$$
u^{1} \leq \alpha<u^{1}+\frac{a N}{2} .
$$

Proof. Consider the function $\psi=\mathbf{c}+\mathbf{e}$. We have two cases.

Case 1. Assume that $\Psi_{+}=0$. Taking $\alpha \equiv u^{1}$ and using that $\mathbf{c}+\mathbf{e} \leq 0$, it follows from (59) that $\alpha$ is a lower solution of (58).
Case 2. Assume that $\Psi_{+}>0$. Let $h_{k}=\psi_{k}^{+} \Psi_{-}-\psi_{k}^{-} \Psi_{+}$. Then using

$$
\sum_{k=2}^{N-1} h_{k}=\sum_{k=2}^{N-1}\left[\psi_{k}^{+} \Psi_{-}-\psi_{k}^{-} \Psi_{+}\right]=0
$$

and [12, Proposition 3], it follows that there exists $\mathbf{w} \in V^{N-2}$ such that

$$
\nabla\left[\phi\left(\Delta w_{k}\right)\right]=h_{k}, \quad w_{1}=w_{N}, \quad \Delta w_{1}=\Delta w_{N-1} .
$$

Let us take $u^{0}=1 / \Psi_{+}$and $\omega_{j}=\min \left\{0, \phi^{-1}\left(\sum_{l=2}^{j-1} u^{0} h_{l}+\right.\right.$ $\left.\left.Q_{\phi}\left(u^{0} \mathbf{h}\right)\right)\right\}$ for $j=3, \ldots, N-1$. Then we define

$$
\begin{aligned}
\alpha_{2}= & u^{1}-\min _{k \in[3, N-1]_{\mathbb{Z}}} \sum_{j=3}^{k} \omega_{j}, \\
\alpha_{k}= & u^{1}+\sum_{j=3}^{k} \phi^{-1}\left(\sum_{l=2}^{j-1} u^{0} h_{l}+Q_{\phi}\left(u^{0} \mathbf{h}\right)\right) \\
& -\min _{k \in[3, N-1]_{\mathbb{Z}}} \sum_{j=3}^{k} \omega_{j}, \quad k \in[3, N-1]_{\mathbb{Z} \cdot}
\end{aligned}
$$

Let $\alpha_{1}=\alpha_{N}=\left(\alpha_{2}+\alpha_{N-1}\right) / 2$; then $\Delta \alpha_{1}=\Delta \alpha_{N-1}$. On the other hand, we have that

$$
\Delta \alpha_{k}=\phi^{-1}\left(\sum_{l=2}^{k} u^{0} h_{l}+Q_{\phi}\left(u^{0} \mathbf{h}\right)\right), \quad 2 \leq k \leq N-1 .
$$

Since $\min _{k \in[3, N-1]_{\mathbb{Z}}} \sum_{j=3}^{k} \omega_{j} \leq 0$, Lemma 6 implies (61). Now, we will show that $\alpha$ is the lower solution of (58). By using (60), it follows that $\Psi_{+} \leq \Psi_{-}$; this together with the definitions of $\alpha, u^{0}$, and $h_{k}$ implies that

$$
\begin{aligned}
\nabla\left[\phi\left(\Delta \alpha_{k}\right)\right] & =u^{0} h_{k}=\frac{1}{\Psi_{+}}\left[\psi_{k}^{+} \Psi_{-}-\psi_{k}^{-} \Psi_{+}\right] \\
& \geq \psi_{k}^{+}-\psi_{k}^{-}=\psi_{k}, \quad k \in[2, N-1]_{\mathbb{Z}} .
\end{aligned}
$$

From (59) and (61), we deduce that

$$
g_{0}\left(k, \alpha_{k}\right)+e_{k} \leq \psi_{k}, \quad \forall k \in[2, N-1]_{\mathbb{Z} \cdot}
$$

Consequently,

$$
\nabla\left[\phi\left(\Delta \alpha_{k}\right)\right] \geq g_{0}\left(k, \alpha_{k}\right)+e_{k}, \quad \forall k \in[2, N-1]_{\mathbb{Z}}
$$

By a similar argument, it is easy to prove the following theorem.

Theorem 11. Suppose that there exist $u^{2}>0$ and $\mathbf{d}=$ $\left(d_{2}, \ldots, d_{N-1}\right) \in \mathbb{R}^{N-2}$ such that

$$
g_{0}(k, u) \geq d_{k}
$$$$
\text { for any }(k, u) \in[2, N-1]_{\mathbb{Z}} \times\left[u^{2}, u^{2}+\frac{a N}{2}\right] \text {. }
$$

If

$$
\overline{\mathbf{d}}+\overline{\mathbf{e}} \geq 0,
$$

then (58) has an upper solution $\beta$ such that

$$
u^{2} \leq \beta<u^{2}+\frac{a N}{2} .
$$




\section{Some Applications for Singular Perturbations Problems}

4.1. Strong Repulsive Perturbations. Consider the discrete periodic problem

$$
\begin{gathered}
\nabla\left[\phi\left(\Delta u_{k}\right)\right]+r_{k} u_{k}-g\left(u_{k}\right)=e_{k}, \\
u_{1}=u_{N}, \quad \Delta u_{1}=\Delta u_{N-1},
\end{gathered}
$$

where $\mathbf{r}=\left(r_{2}, \ldots, r_{N-1}\right), \mathbf{e}=\left(e_{2}, \ldots, e_{N-1}\right) \in \mathbb{R}^{N-2}$, and $g$ : $(0, \infty) \rightarrow \mathbb{R}$ is continuous and satisfies

$$
\lim _{x \rightarrow 0} g(x)=+\infty, \quad \lim _{x \rightarrow \infty} g(x)=0 .
$$

The main result of this subsection is the following theorem.

Theorem 12. Assume that (73) holds. If either

$$
\overline{\mathbf{r}}>0 \quad \text { or } \quad \overline{\mathbf{r}}=0, \quad \overline{\mathbf{e}}<-\frac{a N R_{-}}{2(N-2)},
$$

then problem (72) has at least one solution.

Proof. Notice that from (73) it follows that there exists a constant $\beta$ sufficiently small such that

$$
0 \leq g(\beta)+e_{k}-r_{k} \beta,
$$

which means that $\beta$ is an upper solution of (72).

Now we construct a lower solution of (72) by applying Theorem 10. Consider the continuous function $g_{0}$ : $[2, N-1]_{\mathbb{Z}} \times(0, \infty) \rightarrow \mathbb{R}$ defined by

$$
g_{0}\left(k, u_{k}\right)=-r_{k} u_{k}+g\left(u_{k}\right)
$$

$g^{*}:(0, \infty) \rightarrow \mathbb{R}$ given by

$$
g^{*}(u)=\max _{x \in[u, u+a N / 2]} g(x),
$$

and $\gamma^{*}:(0, \infty) \rightarrow \mathbb{R}$ defined by

$$
\gamma^{*}(u)=-R u+\frac{a N}{2} R_{-}+(N-2) g^{*}(u) .
$$

Case 1. Assume that $\overline{\mathbf{r}}>0$. This together with (73) implies that

$$
\lim _{u \rightarrow \infty} \gamma^{*}(u)=-\infty
$$

so there exists $u^{1}>0$ such that $\gamma^{*}\left(u^{1}\right) \leq-E$. In order to apply Theorem 10, define

$$
c_{k}=r_{k}^{-}\left(u^{1}+\frac{a N}{2}\right)-r_{k}^{+} u^{1}+g^{*}\left(u^{1}\right), \quad k \in[2, N-1]_{\mathbb{Z} \cdot}
$$

It follows that $C=\gamma^{*}\left(u^{1}\right)$ and $C+E \leq 0$, meaning that condition $(60)$ is fulfilled. One has that

$$
\begin{aligned}
g_{0}\left(k, u_{k}\right) & =r_{k}^{-} u_{k}-r_{k}^{+} u_{k}+g\left(u_{k}\right) \\
& \leq r_{k}^{-}\left(u^{1}+\frac{a N}{2}\right)-r_{k}^{+} u^{1}+g^{*}\left(u^{1}\right),
\end{aligned}
$$

for all $\left(k, u_{k}\right) \in[2, N-1]_{\mathbb{Z}} \times\left[u^{1}, u^{1}+a N / 2\right]$. So, condition (59) holds. Then, from Theorem 10 we infer that (72) has a lower solution $\alpha$. Therefore, from Theorem 9, we can obtain the result.

Case 2. Assume that $\overline{\mathbf{r}}=0$ and $\overline{\mathbf{e}}<-a N R_{-} / 2(N-2)$. It follows that

$\gamma^{*}(u)=\frac{a N}{2} R_{-}+(N-2) g^{*}(u), \quad \lim _{u \rightarrow \infty} \gamma^{*}(u)=\frac{a N}{2} R_{-}$.

Then, there exists $u^{1}>0$ such that $\gamma^{*}\left(u^{1}\right) \leq-E$, since $\overline{\mathbf{e}}<$ $-a N R_{-} / 2(N-2)$. The result follows by a similar argument to that used in Case 1.

Remark 13. Theorem 9 in [12] follows from Theorem 12 just taking $r=0$.

Example 14. Consider the repulsive singular periodic problem

$$
\begin{gathered}
\nabla\left(\frac{\Delta u_{k}}{\sqrt{1-\kappa\left(\Delta u_{k}\right)^{2}}}\right)+r_{k} u_{k}-\frac{1}{\left(u_{k}\right)^{\lambda}}=e_{k}, \\
k \in[2, N-1]_{\mathbb{Z}}, \\
u_{1}=u_{N}, \quad \Delta u_{1}=\Delta u_{N-1},
\end{gathered}
$$

where $\mathbf{r}=\left(r_{2}, \ldots, r_{N-1}\right), \mathbf{e}=\left(e_{2}, \ldots, e_{N-1}\right) \in \mathbb{R}^{N-2}$, and $\lambda \geq$ 1. If either $\overline{\mathbf{r}}>0$ or $\overline{\mathbf{r}}=0, \overline{\mathbf{e}}<-N R_{-} / 2 \sqrt{\kappa}(N-2)$, then (83) has at least one solution.

In the case $\mathbf{r}<0$, there exists $s_{0}<0$ such that (83) has at least two solutions provided that $\mathbf{e} \leq s_{0}$ holds true. In fact, in this case, problem (83) has two strict upper solutions $\beta_{1}$, $\beta_{2}>0$ and a strict lower solution $\alpha>0$ such that $\beta_{1}<\alpha<\beta_{2}$. Thus, the result follows from Lemma 4 and Theorem 9 .

4.2. Mixed Singularities. Consider the discrete periodic problem

$$
\begin{gathered}
\nabla\left[\phi\left(\Delta u_{k}\right)\right]+r_{k} u_{k}+g\left(k, u_{k}\right)=e_{k}, \\
u_{1}=u_{N}, \quad \Delta u_{1}=\Delta u_{N-1},
\end{gathered}
$$

where $\mathbf{r}=\left(r_{2}, \ldots, r_{N-1}\right), \mathbf{e}=\left(e_{2}, \ldots, e_{N-1}\right) \in \mathbb{R}^{N-2}$, and $g$ : $[2, N-1]_{\mathbb{Z}} \times(0, \infty) \rightarrow \mathbb{R}$ is continuous and satisfies

$$
\lim _{u \rightarrow \infty} g(k, u)=0, \quad \text { uniformly with } k \in[2, N-1]_{\mathbb{Z}} .
$$

Let the continuous function $g_{0}:[2, N-1]_{\mathbb{Z}} \times(0, \infty) \rightarrow \mathbb{R}$ defined by

$$
g_{0}\left(k, u_{k}\right)=-r_{k} u_{k}-g\left(k, u_{k}\right),
$$

$g_{*}, g^{*}:[2, N-1]_{\mathbb{Z}} \times(0, \infty) \rightarrow \mathbb{R}$ given by

$$
\begin{aligned}
& g_{*}(k, u)=\min _{x \in[u, u+a N / 2]} g(k, x), \\
& g^{*}(k, u)=\max _{x \in[u, u+a N / 2]} g(k, x),
\end{aligned}
$$


and $\gamma_{*}:(a N / 2, \infty) \rightarrow \mathbb{R}, \gamma^{*}:(0, \infty) \rightarrow \mathbb{R}$, defined by

$$
\begin{gathered}
\gamma_{*}(u)=-R u+\frac{a N}{2} R_{+}-\sum_{j=2}^{N-1} g_{*}\left(j, u-\frac{a N}{2}\right), \\
\gamma^{*}(u)=-R u-\frac{a N}{2} R_{+}-\sum_{j=2}^{N-1} g^{*}(j, u) .
\end{gathered}
$$

The following lemma plays a key role to prove the main result in this subsection.

Lemma 15. Let (85) hold and $\gamma_{*}^{m}:=\inf \gamma_{*}$. If $\overline{\mathbf{r}}<0$ and $-E>$ $\gamma_{*}^{m}$, then (84) has at least one solution.

Proof. Since $-E>\gamma_{*}^{m}$, there exists $z>a N / 2$ such that $\gamma_{*}(z) \leq-E$. Let us take $u^{1}=z-a N / 2>0$ and $\mathbf{c}=$ $\left(c_{2}, \ldots, c_{N-1}\right) \in \mathbb{R}^{N-2}$ by

$$
c_{k}=r_{k}^{-}\left(u^{1}+\frac{a N}{2}\right)-r_{k}^{+} u^{1}-g_{*}\left(k, u^{1}\right) .
$$

Then it follows that conditions (59) and (60) hold. Thus, from Theorem 10 we infer that (84) has a lower solution $\alpha$ such that $u^{1} \leq \alpha<u^{1}+a N / 2$.

On the other hand, using the fact that $\overline{\mathbf{r}}<0$, there exists $u^{2} \geq z$ such that $\gamma^{*}\left(u^{2}\right) \geq-E$. Consider $\mathbf{d}=\left(d_{2}, \ldots, d_{N-1}\right) \in$ $\mathbb{R}^{N-2}$ by

$$
d_{k}=r_{k}^{-} u^{2}-r_{k}^{+}\left(u^{2}+\frac{a N}{2}\right)-g^{*}\left(k, u^{2}\right) .
$$

Then, it follows that conditions (69) and (70) hold. Therefore, from Theorem 11 we can get that (84) has an upper solution $\beta$ such that $\alpha \leq \beta$. The result follows from Lemma 4 .

Remark 16. From Lemma 15, the solution $\mathbf{u}$ of (84) is a positive solution since $0<u^{1} \leq \alpha \leq \mathbf{u} \leq \beta<u^{2}+a N / 2$.

Let us consider the discrete periodic problem

$$
\begin{gathered}
\nabla\left[\phi\left(\Delta u_{k}\right)\right]+r_{k} u_{k}+\frac{m_{k}}{\left(u_{k}\right)^{\lambda}}=e_{k}, \quad k \in[2, N-1]_{\mathbb{Z}} \\
u_{1}=u_{N}, \quad \Delta u_{1}=\Delta u_{N-1},
\end{gathered}
$$

where $\mathbf{r}=\left(r_{2}, \ldots, r_{N-1}\right), \mathbf{m}=\left(m_{2}, \ldots, m_{N-1}\right), \mathbf{e}=$ $\left(e_{2}, \ldots, e_{N-1}\right) \in \mathbb{R}^{N-2}$, and $\lambda>0$. We have the following theorem.

Theorem 17. If $\overline{\mathbf{r}}<0$ and

$$
\begin{aligned}
-E> & (1+\lambda)\left[\frac{|R|^{\lambda} M_{-}}{\lambda^{\lambda}}\right]^{1 /(1+\lambda)} \\
& +\frac{a N}{2} R_{-}-M_{+}\left[\frac{a N}{2}+\left(\frac{\lambda M_{-}}{|R|}\right)^{1 /(1+\lambda)}\right]^{-\lambda}
\end{aligned}
$$

then (91) has at least one solution.
Proof. We have divided two cases.

Case 1. Assume that $M_{-}=0$. In this case one has that

$$
\gamma_{*}(u)=-R u+\frac{a N}{2} R_{+}-\frac{M_{+}}{u^{\lambda}},
$$

implying that $\gamma_{*}^{m}=\gamma_{*}(a N / 2)$. So, (92) becomes $-E>\gamma_{*}^{m}$, and the result follows from Lemma 15.

Case 2. Assume that $M_{-}>0$. Notice that the minimum of $u \mapsto-R u+(a N / 2) R_{+}+M_{-} /(u-a N / 2)^{\lambda}$ is attained in $u^{0}=$ $a N / 2+\left[\lambda M_{-} /|R|\right]^{1 /(\lambda+1)}$ and

$$
\gamma_{*}\left(u^{0}\right) \leq-R u^{0}+\frac{a N}{2} R_{+}+\frac{M_{-}}{\left(u^{0}-a N / 2\right)^{\lambda}}-\frac{M_{+}}{\left(u^{0}\right)^{\lambda}} .
$$

It is not difficult to verify that $\gamma_{*}\left(u^{0}\right)<-E$ by using (92). Hence, $-E>r_{*}^{m}$, and the result follows from Lemma 15 .

Example 18. Consider the discrete periodic problem with repulsive singularity:

$$
\begin{gathered}
\nabla\left(\frac{\Delta u_{k}}{\sqrt{1-\kappa\left(\Delta u_{k}\right)^{2}}}\right)+r_{k} u_{k}-\frac{m_{k}}{\left(u_{k}\right)^{\lambda}}=e_{k}, \\
k \in[2, N-1]_{\mathbb{Z}}, \\
u_{1}=u_{N}, \quad \Delta u_{1}=\Delta u_{N-1},
\end{gathered}
$$

where $\mathbf{r}=\left(r_{2}, \ldots, r_{N-1}\right), \mathbf{m}=\left(m_{2}, \ldots, m_{N-1}\right)$, and $\mathbf{e}=$ $\left(e_{2}, \ldots, e_{N-1}\right) \in \mathbb{R}^{N-2}$ with $\mathbf{m} \geq 0$ and $\lambda>0$. If $\overline{\mathbf{r}}<0$ and

$$
-E>(1+\lambda)\left[\frac{|R|^{\lambda} M}{\lambda^{\lambda}}\right]^{1 /(1+\lambda)}+\frac{N R_{-}}{2 \sqrt{\kappa}},
$$

then the above problem has at least one solution.

Example 19. Consider the periodic problem with attractive singularity

$$
\begin{gathered}
\nabla\left(\frac{\Delta u_{k}}{\sqrt{1-\kappa\left(\Delta u_{k}\right)^{2}}}\right)+r_{k} u_{k}+\frac{m_{k}}{\left(u_{k}\right)^{\lambda}}=e_{k}, \\
k \in[2, N-1]_{\mathbb{Z}}, \\
u_{1}=u_{N}, \quad \Delta u_{1}=\Delta u_{N-1},
\end{gathered}
$$

where $\mathbf{r}=\left(r_{2}, \ldots, r_{N-1}\right), \mathbf{m}=\left(m_{2}, \ldots, m_{N-1}\right)$, and $\mathbf{e}=$ $\left(e_{2}, \ldots, e_{N-1}\right) \in \mathbb{R}^{N-2}$ with $\mathbf{m} \geq 0$ and $\lambda>0$. If $\overline{\mathbf{r}}<0$ and

$$
E<M\left(\frac{2 \sqrt{\kappa}}{N}\right)^{\lambda}-\frac{N R_{-}}{2 \sqrt{\kappa}}
$$

then the above problem has at least one solution.

In connection with Example 19, if $\mathbf{r}=0$, then we have the following theorem. 
Theorem 20. Consider the discrete periodic problem with attractive singularity

$$
\begin{gathered}
\nabla\left[\phi\left(\Delta u_{k}\right)\right]+\frac{m_{k}}{\left(u_{k}\right)^{\lambda}}=e_{k}, \quad k \in[2, N-1]_{\mathbb{Z}}, \\
u_{1}=u_{N}, \quad \Delta u_{1}=\Delta u_{N-1},
\end{gathered}
$$

where $\mathbf{m}=\left(m_{2}, \ldots, m_{N-1}\right), \mathbf{e}=\left(e_{2}, \ldots, e_{N-1}\right) \in \mathbb{R}^{N-2}$ with $\mathbf{m} \geq 0$ and $\lambda>0$. If

$$
0<E<M\left(\frac{2}{a N}\right)^{\lambda},
$$

then (99) has at least one solution.

Proof. We will use the same strategy as in the proof of Lemma 15. In this case one has that $g_{0}(k, u)=-m_{k} / u^{\lambda}$.

Clearly, it follows from (100) that there exists $z>a N / 2$ such that $E<M z^{-\lambda}$. Let us define $u^{1}=z-a N / 2>0$ and $\mathbf{c}=\left(c_{2}, \ldots, c_{N-1}\right) \in \mathbb{R}^{N-2}$ by

$$
c_{k}=-m_{k}\left(u^{1}+\frac{a N}{2}\right)^{-\lambda} .
$$

Then, it follows that conditions (59) and (60) hold. Therefore, from Theorem 10 we infer that (99) has a lower solution $\alpha$ such that $u^{1} \leq \alpha<u^{1}+a N / 2$.

Using again (100), it follows that there exists $u^{2}>z$ such that $E \geq M\left(u^{2}\right)^{-\lambda}$. Let us define $\mathbf{d}=\left(d_{2}, \ldots, d_{N-1}\right) \in \mathbb{R}^{N-2}$ by $d_{k}=-m_{k}\left(u^{2}\right)^{-\lambda}$. Then it follows that conditions (69) and (70) are true. Hence, from Theorem 11 we infer that (99) has an upper solution $\beta$ such that $u^{2} \leq \beta<u^{2}+a N / 2$.

Consequently, (99) has a lower solution $\alpha$ and an upper solution $\beta$ such that $\alpha \leq \beta$. The result follows from Lemma 4 .

In the "pure" attractive case we have the following result concerning (84).

Theorem 21. Assume that (85) and

$$
\lim _{u \rightarrow 0} g(k, u)=+\infty, \quad \text { uniformly with } k \in[2, N-1]_{\mathbb{Z}}
$$

hold. Then (84) has at least one solution provided that either

$$
\overline{\mathbf{r}}<0 \quad \text { or } \quad \overline{\mathbf{r}}=0, \quad E>\frac{a N}{2} R_{+} .
$$

Proof. Notice that from (102) it follows that any sufficiently small positive constant $\alpha$ is a lower solution of (84). The construction of an upper solution $\beta \geq \alpha$ for (84) is similar as in Lemma 15. Then the result follows from Lemma 4.

Remark 22. Theorem 21 follows from [12] taking $\mathbf{r}=0$ in Theorem 21.

Example 23. Let us consider again problem (97), with the condition $\mathbf{m}>0$. If either $\overline{\mathbf{r}}<0$ or $\overline{\mathbf{r}}=0$ and $E>N R_{+} / 2 \sqrt{\kappa}$, then (97) has at least one solution.
Example 24. Let $\lambda>0, \mu \geq 1$ with $\mu>\lambda$ and consider the discrete periodic problem

$$
\begin{gathered}
\nabla\left(\frac{\Delta u_{k}}{\sqrt{1-\left(\Delta u_{k}\right)^{2}}}\right)+c \frac{\Delta u_{k}}{\left(u_{k}\right)^{4 / 5}}+\frac{1}{\left(u_{k}\right)^{\lambda}}-\frac{1}{\left(u_{k}\right)^{\mu}}=e_{k}\left(u_{k}\right)^{\lambda}, \\
u_{1}=u_{N}, \quad \Delta u_{0}=\Delta u_{N-1},
\end{gathered}
$$

where $\mathbf{e}=\left(e_{2}, \ldots, e_{N-1}\right) \in \mathbb{R}^{N-2}$ with $\mathbf{e} \leq 0$. It is not difficult to prove that the above problem has at least one solution for any $c \in \mathbb{R}$ by using Theorem 9 .

\section{Conflict of Interests}

The authors declare that they have no conflict of interests.

\section{Authors' Contribution}

Ruyun Ma completed the main study, carried out the results of this paper, and drafted the paper. Yanqiong Lu checked the proofs and verified the calculation. All the authors read and approved the final paper.

\section{Acknowledgments}

The authors are very grateful to the anonymous referees for their valuable suggestions. This work was supported by the NSFC (no. 11361054), SRFDP (no. 20126203110004), and Gansu provincial National Science Foundation of China (no. 1208RJZA258).

\section{References}

[1] W. Grenier, Classical Mechanics-Point Particles and Relativity, Springer, Berlin, Germany, 2004.

[2] E. H. Hutten, "Relativistic (non-linear) oscillator," Nature, vol. 205, p. 892, 1965.

[3] L. A. MacColl, "Theory of the relativistic oscillator," American Journal of Physics, vol. 25, pp. 535-538, 1957.

[4] D. D. Hai, K. Schmitt, and R. Shivaji, "Positive solutions of quasilinear boundary value problems," Journal of Mathematical Analysis and Applications, vol. 217, no. 2, pp. 672-686, 1998.

[5] C. Bereanu and J. Mawhin, "Existence and multiplicity results for some nonlinear problems with singular $\phi$-Laplacian," Journal of Differential Equations, vol. 243, no. 2, pp. 536-557, 2007.

[6] C. Bereanu, D. Gheorghe, and M. Zamora, "Periodic solutions for singular perturbations of the singular $\varphi$-Laplacian operator," Communications in Contemporary Mathematics, vol. 15, no. 4, 1250063, 22 pages, 2013.

[7] C. Bereanu, D. Gheorghe, and M. Zamora, "Non-resonant boundary value problems with singular $\phi$-Laplacian operators," Nonlinear Differential Equations and Applications, vol. 20, no. 3, pp. 1365-1377, 2013.

[8] C. Bereanu, P. Jebelean, and P. J. Torres, "Positive radial solutions for Dirichlet problems with mean curvature operators in Minkowski space," Journal of Functional Analysis, vol. 264, no. 1, pp. 270-287, 2013. 
[9] W. G. Kelley and A. C. Peterson, Difference Equations: An Introduction with Applications, Academic Press, New York, NY, USA, 1991.

[10] R. P. Agarwal, Difference Equations and Inequalities, Marcel Dekker, New York, NY. USA, 2nd edition, 2000.

[11] C. Bereanu and J. Mawhin, "Existence and multiplicity results for nonlinear second order difference equations with Dirichlet boundary conditions," Mathematica Bohemica, vol. 131, no. 2, pp. 145-160, 2006.

[12] C. Bereanu and J. Mawhin, "Boundary value problems for second-order nonlinear difference equations with discrete $\phi$ Laplacian and singular $\phi$," Journal of Difference Equations and Applications, vol. 14, no. 10-11, pp. 1099-1118, 2008.

[13] C. Bereanu and H. B. Thompson, "Periodic solutions of second order nonlinear difference equations with discrete $\phi$-Laplacian," Journal of Mathematical Analysis and Applications, vol. 330, no. 2, pp. 1002-1015, 2007.

[14] R. Ma, Y. Lu, and T. Chen, "Existence of one-signed solutions of discrete second-order periodic boundary value problems," Abstract and Applied Analysis, vol. 2012, Article ID 437912, 13 pages, 2012.

[15] R. Ma and Y. Lu, "Positive periodic solutions of secondorder difference equations with weak singularities," Advances in Difference Equations, vol. 2012, article 90, 11 pages, 2012.

[16] Y. Lu and R. Ma, "On positive periodic solutions of secondorder difference equations with attractive-repulsive singularities," Advances in Difference Equations, vol. 2012, article 186, 9 pages, 2012.

[17] Y. Lu and R. Ma, "Reverse-order lower and upper functions for periodic problems of second-order singular difference equations," Abstract and Applied Analysis, vol. 2013, Article ID 176465, 5 pages, 2013.

[18] R. P. Agarwal and P. J. Y. Wong, Advanced Topics in Difference Equations, vol. 404 of Mathematics and Its Applications, Kluwer Academic Publishers, Dordrecht, The Netherlands, 1997. 


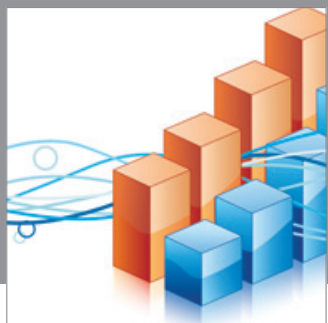

Advances in

Operations Research

mansans

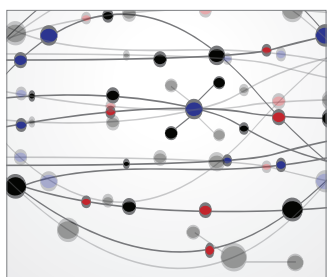

The Scientific World Journal
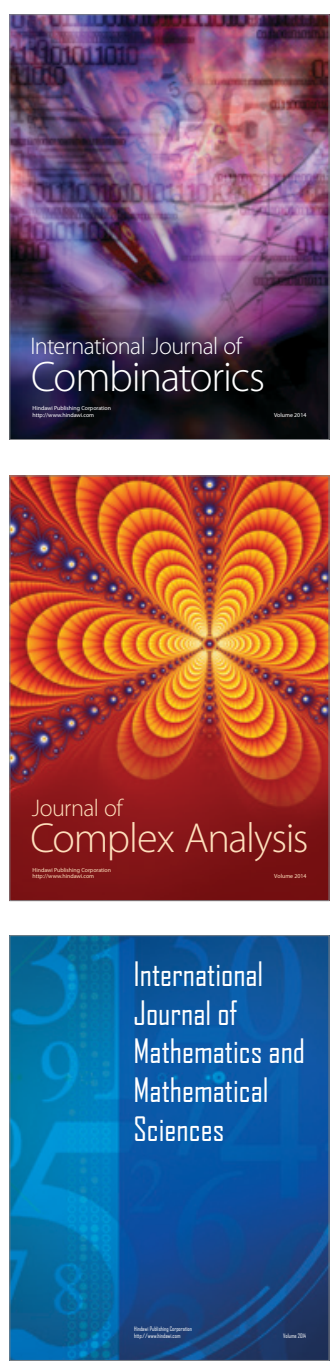
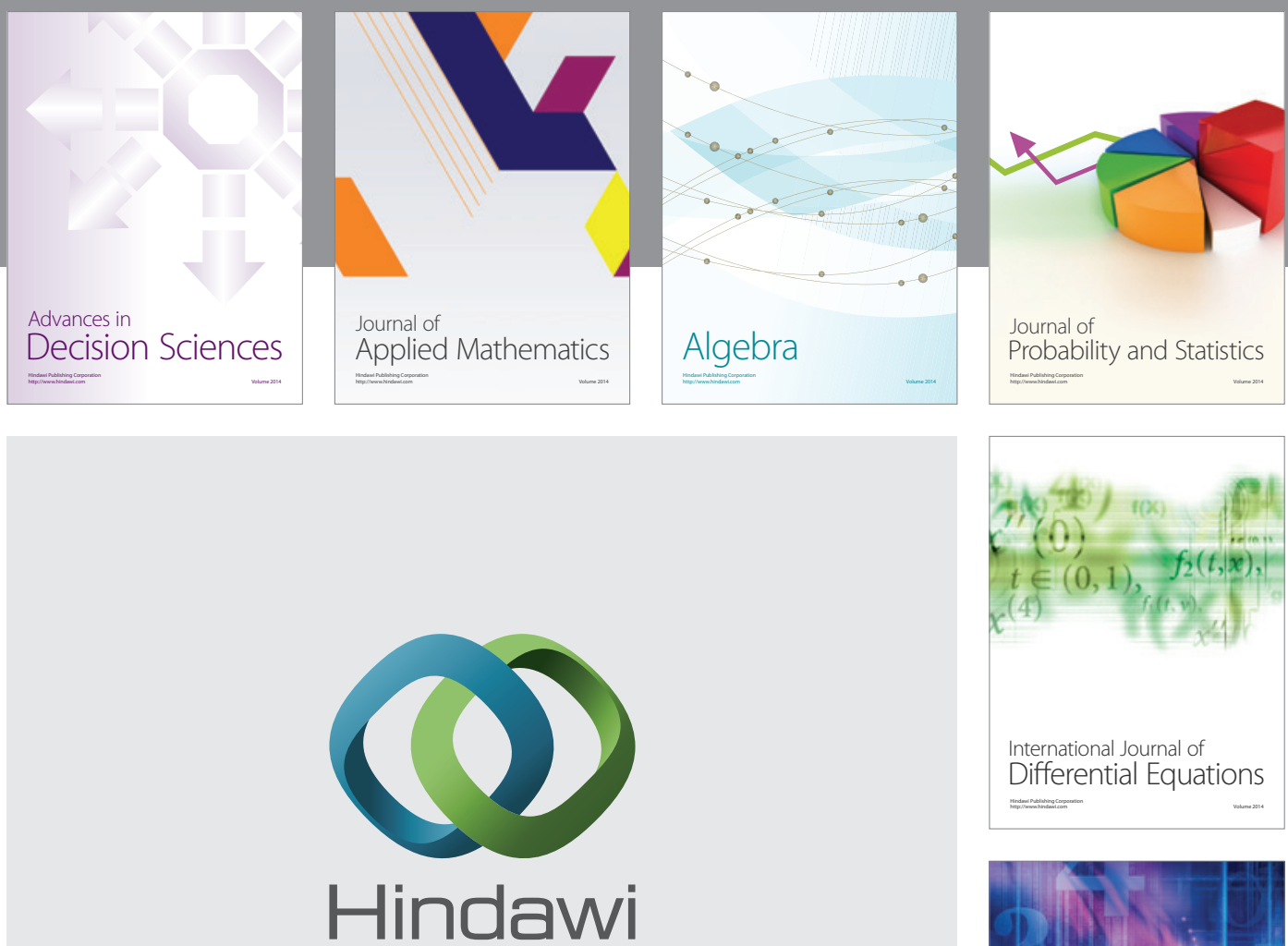

Submit your manuscripts at http://www.hindawi.com
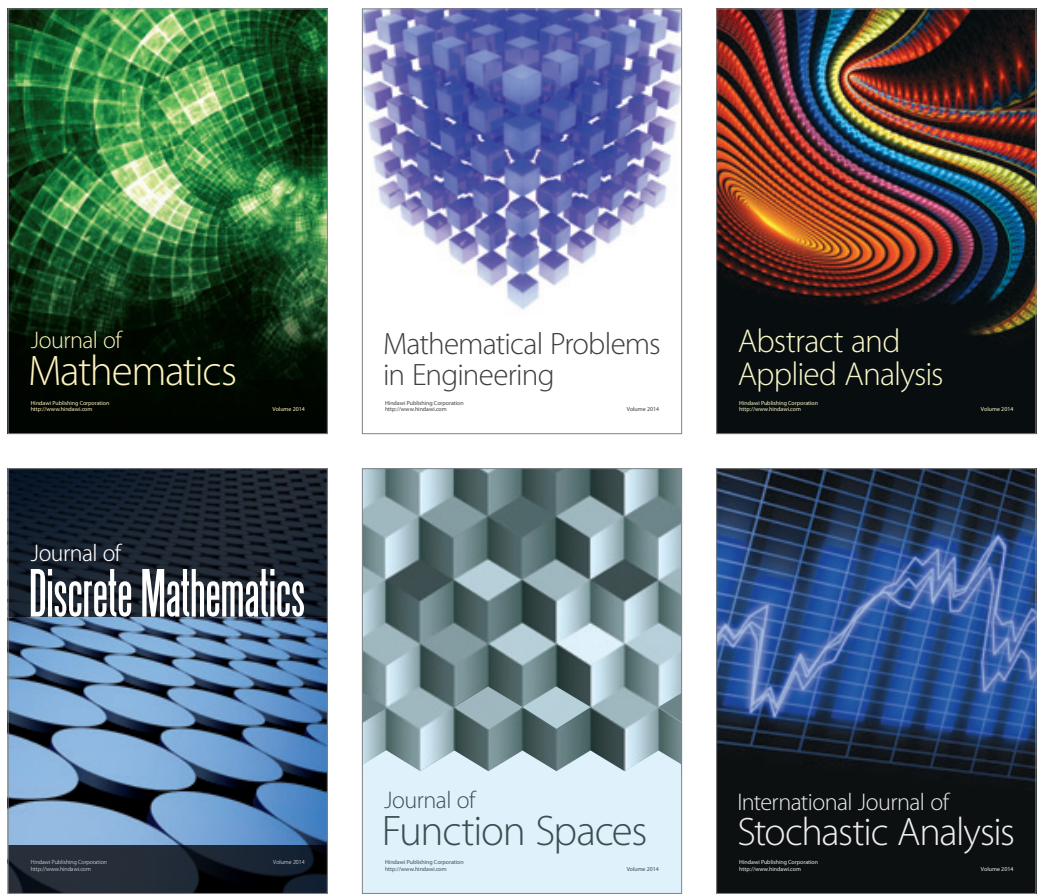

Journal of

Function Spaces

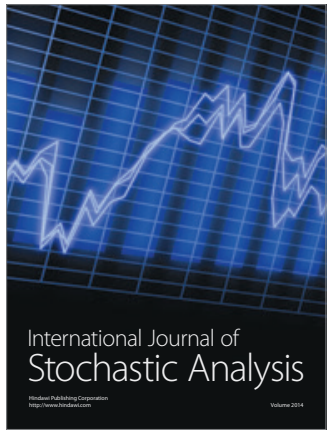

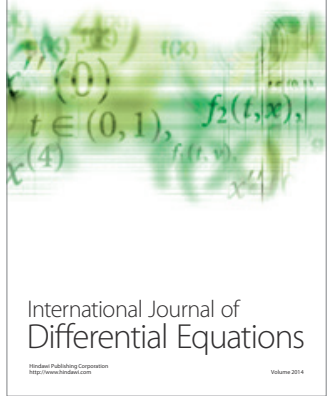
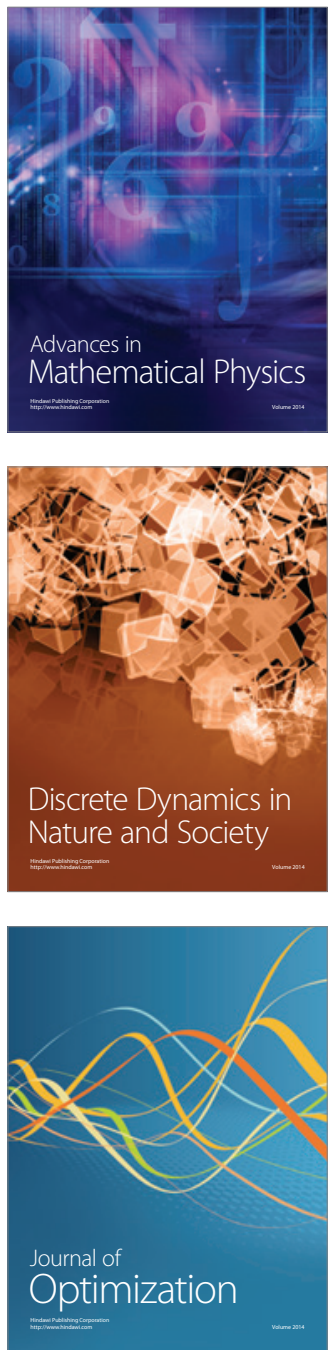